\title{
SOBRE LAS CAUSAS \\ DE LA SUSTITUCIÓN \\ DE CANTARE HABEO POR CANTARÉ
}

\author{
Witold Sobczak*
}

\begin{abstract}
Astract. The aim of the article is to comment on the reasons for the replacement of the analytic future form (cantare habeo) by the synthetic one (cantaré) in Medieval Spanish. Undoubtedly, we are dealing with a process considered to be a linguistic change, which was triggered not only by a series of different morphological and phonological factors, but also by those of a semantic and syntactic nature. We should nonetheless bear in mind that this replacement basically meant a change in the formal expression of posteriority. For this reason, its creation did not prove to be so innovative, unlike, for example, the appearance of cantaría, which was a completely new and nonexistent conjugation verbal category in Latin.
\end{abstract}

Keywords: synthetic future, analytic future, posteriority, linguistic change, Medieval Spanish.

Palabras clave: futuro sintético, futuro analítico, posterioridad, cambio lingüístico, español medieval.

Como ya se ha indicado en varias ocasiones, el reemplazo del futuro sintético por el analítico (también llamados morfológico y perifrástico, respectivamente) documentado en el español contemporáneo, que constituye un tema clásico en la lingüística

\footnotetext{
* Universidad de Łódź, Facultad de Filología, Departamento de Filología Española, c/ Pomorska 171/173, 90-236 Łódź, Polonia, witeksobczak87@gmail.com.
} 
románica, no es absolutamente nada nuevo y tiene carácter cíclico, al menos en las lenguas procedentes del latín. Si analizamos más detalladamente la evolución de las formas encargadas de denotar posterioridad a partir del latín clásico hasta el castellano actual, veremos que se repite un esquema según el cual la expresión de lo posterior, una vez hecha analítica, pasa a sintetizarse y, como consecuencia de ello, a caer en desuso a favor de otra más analítica que va ocupando paulatinamente su lugar. Teniendo en cuenta que la rivalidad entre las formas sintética y analítica se remonta al latín, el objeto de nuestro trabajo será comentar las causas principales del reemplazo del futuro analítico (cantare habeo) por la forma sintética (cantaré).

En primer lugar, conviene recordar que la descomposición del Imperio Romano en el siglo $\mathrm{V}$ y el aislamiento de las provincias convertidas en estados bárbaros abrió camino a numerosas innovaciones que tocaron prácticamente a todos los niveles de la lengua desde la fonética hasta las preferencias por el vocabulario (Lapesa, 1988 [1942]: 83). A partir del momento en el que los constituyentes del nuevo futuro, íntimamente fusionados, formaron un conjunto, el verbo habeo (el más utilizado en la Romania como generador de perífrasis de futuro) fue perdiendo tanto su autonomía fónica como semántica y funcional, lo cual insinuaba su transformación en una nueva forma sintética (Lleal, 1990: 91). Así, cantare habeo procedente del latín vulgar, denominado en la tradición gramatical como "futuro analítico", fue desapareciendo poco a poco hasta llegar a convertirse en una forma fija: cantaré (Company Company, 2006: 356).

En lo referente a la forma sintética del futuro, cabe destacar que el primer ejemplo irrefutable del futuro románico, comúnmente aceptado por los estudiosos de este tema, aparece en una compilación histórica francesa del año 613 titulada Crónica de Fredigarius (Lleal, 1990: 91; Andres Suárez, 1994: 98; Company Company, 2006: 356; Veiga, 2011: 307). Es de subrayar que los testimonios para otras lenguas románicas son considerablemente más tardíos. En el caso del castellano, la primera obra donde se registran los futuros perfectamente formados, con cambios secundarios e interpolación de pronombres átonos (nefrgarsan "se 
ahogarán"), son las Glosas Emilianenses fechadas habitualmente en la segunda mitad del siglo XI ${ }^{1}$ (Bustos Tovar, 2004: 301).

A la hora de hablar de las causas de la sustitución del futuro analítico latino por el sintético medieval, hemos de tener muy en cuenta que una de las peculiaridades de la forma cantare habeo radicaba en que solo se admitían uno u ocasionalmente dos clíticos pronominales ${ }^{2}$ colocados entre el infinitivo y haber (Penny, 1993: 138; Andres Suárez, 1994: 110-111). La interposición en cuestión hacía que el infinitivo mantuviese su estructura fonológica, de modo que el futuro analítico ofrecía una forma estructural y una colocación fijas conforme con el siguiente esquema: infinitivo-clítico-infinitivo. Tal solución era muy común y gozaba de enorme vitalidad entre los dialectos iberolatinos.

Como advierte Company Company (2006: 386), la forma analítica empleada para denotar lo venidero también presentaba algunas restricciones con respecto a la concurrencia con negación, es decir, se admitían las secuencias negación-clítico-verbo o clítico-negación-verbo, pero nunca negación-verbo-clítico. Además, la forma en cuestión se veía restringida por la persona del clítico, su caso, su anclaje referencial y el comportamiento del sujeto (Company Company, 2006: 387-392). Sin lugar a dudas, el orden obligatorio infinitivo-clítico-auxiliar permite clasificar esta construcción como altamente gramaticalizada en la que el infinitivo cumplía la función de un lexema encargado de aportar el contenido léxico, las formas de haber constituían el auxiliar en la frase y el clítico era un caracterizador, un definidor de la frase (Company Company, 2006: 381).

Al comentar las diferencias entre la forma morfológica y la perifrástica, es de resaltar que el nuevo futuro se caracterizaba por mayor flexibilidad que su correspondiente analítico creado en

1 Como destaca Bustos Tovar (2004: 270), se ha retrasado últimamente esta datación.

2 Según hemos conseguido observar, hay varias hipótesis con respecto a la razón de interponer un clítico entre infinitivo y auxiliar, véase, por ejemplo, Andres Suárez (1994: 110) y Company Company (2006: 380-381). 
latín. Dicha flexibilidad se manifestaba tanto en su distribución como en la selección de clíticos y sujetos. Como apunta Andres Suárez (1994: 110), una de las diferencias más importantes consistía en que en la forma sintética los pronombres personales átonos podían encontrarse en tres posiciones: 1) interpolados, 2) antepuestos y separados del verbo, o 3) pospuestos y fundidos con el verbo. En cambio, en el caso de la forma analítica, como ya se ha dicho, se aceptaba exclusivamente su interpolación.

A juicio de Menéndez Pidal (1989 [1904]: 324), Cano Aguilar (1987: 248) y Penny (1993: 138, 195), la interposición de uno o más pronombres fue posible hasta el siglo XVII, es decir, hasta el momento en el que desaparecieron las formas escindidas y el comienzo de una cláusula estaba representado por formas con un único acento como harélo, doblaréos.

A pesar de que el futuro analítico era productivo en la Edad Media, su empleo quedó considerablemente delimitado por numerosas restricciones distribucionales que regían su uso y hacían que presentara una sintaxis altamente especializada. Como destacan Andres Suárez (1994: 112) y Company Company (2006: 383), desde el punto de vista sintáctico, cantare habeo, que ocupaba preferentemente la posición inicial en la oración, se utilizaba exclusivamente en las oraciones principales, independientes o compuestas de muy bajo grado de subordinación. En cuanto a estas últimas, se trataba de oraciones subordinadas que se integraban en oraciones compuestas con un bajo grado de relacionalidad o cohesión donde cada oración establecía un miembro independiente y correlativo del otro miembro oracional, tales como las condicionales. En realidad, el uso del futuro analítico en la apódosis de las oraciones condicionales parecía ser el rasgo más prominente de su distribución oracional. Otros tipos de oraciones también frecuentes para esta forma eran subordinadas adverbiales, temporales, finales y, en menor medida, concesivas.

La forma cantará, a su vez, era indiferente para la distribución oracional con lo cual podía emplearse con éxito tanto en las oraciones principales como en las subordinadas con mayor grado de complejidad estructural. Como advierten García de Diego (1981 [1951]: 371), Cano Aguilar (1987: 161) y Andres Suárez (1994: 
176-187), la forma sintética del futuro se usaba, entre otros, en la prótasis de las oraciones condicionales, en las temporales y subordinadas: "Si querrás serás querido" (Santillana, p. 29, apud García de Diego, 1981 [1951]: 371), “A la mañana, quando los gallos cantarán” (Cid, 316, apud García de Diego, 1981 [1951]: 371), “miedo han que y verna" (PCid, apud Cano Aguilar, 1987: 161). Según señala Lleal (1990: 246), a partir del siglo XIII el futuro aparecía casi siempre en forma sintética, mientras que los raros ejemplos del futuro analítico reflejaban la conservación de cierto valor de perífrasis de obligación.

Está comprobado que las formas del futuro se actualizan a lo largo de toda la Edad Media, sean soldadas, sean escindidas. Aunque muchos estudiosos hablan de formas sintética y analítica, llamando la atención sobre las semejanzas y diferencias entre ellas, cabe destacar que algunos lingüistas postulan que se trata de una sola forma cuya escisión está relacionada con la inserción de un pronombre personal clítico cuando los factores sintácticos requieren su posposición al verbo. En cambio, si no hay pronombre clítico o cuando este precede al verbo, es obligatorio emplear la variante soldada (Eberenz, 2004: 622). A juicio de Company Company (2006: 363), la variante analítica constituye en realidad un estado evolutivo menos avanzado o arcaico en el proceso de gramaticalización, que originó la nueva forma de denotar posterioridad en las lenguas románicas.

Por lo que se refiere a la creación del futuro sintético, hemos de tener presente que estamos ante un proceso complejo que radicó en una serie de transformaciones fónicas que descompusieron no solo la estructura fonológica del infinitivo, sino también la del verbo haber, lo cual provocó que este perdiera su autonomía semántica, sintáctica y morfológica, y pasara de integrar una perífrasis, una frase verbal, a constituir una única forma simple. En otras palabras, el cambio más notable en la formación del futuro romance fue el hecho de que el infinitivo y el auxiliar haber se convirtieron en dos morfemas, radical y flexivo, respectivamente, de un solo verbo (Company Company, 2006: 371).

Sin entrar en los detalles de la cuestión, conviene observar que el proceso de la síntesis del futuro medieval consistió básicamen- 
te en un conjunto de asimilaciones, tanto regresivas como progresivas, en pérdida de segmentos vocálicos, en resilabificación y redefinición de los límites morfológicos originarios. Todo ello condujo, por un lado, a la fusión entre los dos elementos integrantes de la perífrasis y, por otro, a la fosilización de la construcción originaria (Company Company, 2006: 372). Uno de los aspectos más destacados de este proceso lo constituyó evidentemente la caída de la vocal pretónica /e/ o /i/ en los verbos acabados en -er e -ir, lo que se realizó de modo casi sistemático desde épocas muy tempranas (Cano Aguilar, 1987: 157; Eberenz, 2004: 623; Company Company, 2006: 375). Como indica Penny (1993: 196), de este modo se produjo la contracción de la raíz verbal:

\section{DEBERE HABEO > devér hé > deveré > devré}

Curiosamente, desde los finales del siglo XIV hasta el siglo XVI observamos la reposición de la vocal pretónica y empiezan a documentarse las formas modernas: arrepentiré, conseguiré, sentiré, etc. Es obvio que todo ello contribuyó a la aparición de un gran número de duplicidades que hubieron de sufrir reajustes, por ejemplo, comeré-combré, feriré-ferré (Lapesa, 1988 [1942]: 210). Según apunta Eberenz (2004: 623), la norma literaria empezó a decantarse más decididamente por una forma que por la otra a finales de la Edad Media.

No cabe la menor duda de que el proceso de síntesis nunca fue totalmente regular, sino que más bien la creación del futuro sintético se caracterizó por un gran polimorfismo desde las primeras documentaciones de futuros romances hasta el siglo XVII. A pesar de que "la creación del futuro romance fue una innovación, no conllevó ninguna transformación sustancial en el panorama de la conjugación latina, ya que solo significó un cambio en la expresión formal de una categoría ya existente" (Company Company, 2006: 350) y, por eso, no sorprende el hecho de que Benveniste (1968, apud Company Company, 2006: 350) describiera ese proceso como una "transformación conservadora". En cambio, la creación del pospretérito supuso una transformación innovadora, porque se creó una categoría verbal nueva, inexistente como for- 
ma verbal en la conjugación latina (Benveniste, 1968, apud Company Company, 2006: 350; Veiga, 2011: 299).

En lo tocante al futuro sintético en la Edad Media, también conviene repararse en sus valores temporales y modales. Como es de suponer, además de denotar posterioridad con respecto al origen: "Vientos ferran en las uelas del nauio et te leuaran por la mar" (Primera Crónica General, apud Cano Aguilar, 1987: 160), lo cual constituía su función principal, la forma sintética también se empleaba para expresar probabilidad / conjetura en el momento del habla: "Non será, diz Antioco, en tal logar alçado / que de mi lo defienda, yermo nin poblado" (Libro de Apolonio, apud Cano Aguilar, 1987: 160). Dicho de otro modo, el futuro sintético cantaré en la época medieval se sometía al mecanismo de dislocación temporal descrito detalladamente por Rojo y Veiga (1999). Amén de ello, la forma en cuestión era capaz de expresar la obligación de llevar a cabo algún hecho o un mandato (el futuro de imperativo) e incluso podía expresar acciones atemporales, sin un tiempo específico al utilizarse en textos morales en que se daban consejos para la buena conducta humana (Company Company, 2006: 407). En cambio, en la forma analítica del futuro era constante un valor modal de obligatoriedad originado en el auxiliar haber. En opinión de Company Company (2006: 408), ese matiz parecía estar presente incluso en las oraciones que contenían marcas formales tales como adverbios o coordinación con un futuro sintético simple. Dada la omnipresencia de un valor modal de obligatoriedad, no es de extrañar que, a diferencia de su correspondiente sintético, el futuro analítico fuera incapaz de expresar incertidumbre / duda o incluso el valor atemporal de verdades universales, lo que supone que la forma analítica del futuro no experimentaba la dislocación temporal.

A modo de resumir todo lo que se ha dicho hasta ahora, quisiéramos señalar de nuevo que "la desaparición del futuro latino y la creación de una nueva forma para la expresión del porvenir en las lenguas romances fue un ejemplo clásico de cambio lingüístico multicausal" (Company Company, 2006: 352). La aparición de la forma cantaré constituyó el fruto de motivaciones fonológicas, morfológicas y semánticas que, sin duda alguna, interactuaron 
y presionaron de manera conjunta la generación de la nueva forma. Aunque las formas en cuestión compartían el mismo origen, en pocos aspectos gramaticales el futuro analítico equivalía al sintáctico. La sintaxis restringida que poseía el analítico estaba relacionada con su especialización para indicar topicalidad, es decir, tanto tópicos oracionales, como la topicalidad y la prominencia de ciertos constituyentes (Company Company, 2006: 410). El futuro sintético, a su vez, era una forma ni marcada ni especializada para procesos de topicalización, abarcando tanto la sintaxis propia del futuro analítico, así como otros ámbitos sintácticos. Además, a diferencia de la forma analítica, la sintética era capaz de expresar probabilidad / incertidumbre con respecto al origen, propiedad que refuerza considerablemente su posición en el sistema temporal del castellano actual.

\section{Bibliografía}

Andres Suárez, I. (1994): El verbo español. Sistemas medievales y sistema clásico. Madrid: Gredos.

Benveniste, E. (1968): "Mutations of linguistic categories". En: W. P. Lehmann, Y. Malkiel (eds.), Directions for historical linguistics: A symposium. Austin: Texas University Press, 80-94.

Bustos Tovar, J. J. de (2004): “Las Glosas Emilianenses y Silenses”. En: R. Cano Aguilar (coord.), Historia de la lengua española. Barcelona: Ariel, 291-307.

Cano Aguilar, R. (1988): El español a través de los tiempos. Madrid: Arco/Libros S.A.

Company Company, C. (2006): "Tiempos de formación romance II. Los futuros y condicionales”. En: C. Company Company (coord.), Sintaxis histórica de la lengua española. Primera parte: la frase verbal. México: Fondo de Cultura Económica, 349-418.

Eberenz, R. (2004): “La transición léxica en el español bajomedieval”. En: R. Cano Aguilar (coord.), Historia de la lengua española. Barcelona: Ariel: 643-656.

García de Diego, V. (1981 [1951]): Gramática histórica española. Madrid: Gredos. 
Lapesa, R. (1988 [1942]): Historia de la lengua española. Madrid: Gredos.

Lleal, C. (1990): La formación de las lenguas peninsulares. Barcelona: Barcanova.

Menéndez Pidal, R. (1989 [1904]): Manual de gramática histórica española. Madrid: Espasa-Calpe.

Penny, R. (1993): Gramática histórica del español. Barcelona: Ariel Lingüística. Rojo, G., Veiga, A. (1999): "El tiempo verbal. Los tiempos simples". En: I. Bosque, V. Demonte (eds.), Gramática descriptiva de la lengua española, t. 2. Madrid: Espasa-Calpe, 2868-2934.

Veiga, A. (2011): "Algo más sobre la doble organización temporal en la diacronía del sistema verbal español”. En: C. Sinner, J. L. Ramírez Luengo, M. J. Torrens Álvarez (coords.), Tiempo, espacio y relaciones espacio-temporales desde la perspectiva de la lingüística histórica. San Millán de la Cogolla: Cilengua, 297-330. 